\title{
Kutane Metastasen - Klinik, Histopathologie und spezielle Marker
}

R. Moll

\author{
Cutaneous Metastases - Clinical Presentation, Histopathology and \\ Specific Markers
}

\section{Zusammenfassung}

Tumoren an der Haut stellen ein wichtiges Teilgebiet der Dermatologie dar und umfassen eigentliche Hauttumoren, aber auch Metastasen von Malignomen innerer Organe, deren Diagnose wichtig ist. In der vorliegenden Arbeit werden Klinik und Histopathologie zusammen mit der immunhistochemischen Differenzialdiagnose kurz vorgestellt.

Ca. 2 - $5 \%$ aller Malignom-Fälle führen zu Hautmetastasen, meist erst im Spätstadium. Bei weitem am häufigsten sind Mammakarzinome (bei Patientinnen) gefolgt von Lungenkarzinom, kolorektalem Karzinom, Ovarialkarzinom, malignem Melanom, KopfHals-Karzinom, Nierenzellkarzinom und dem Magenkarzinom. Metastasen an der Haut sind bevorzugt am behaarten Kopf, Thorax und der Nabelregion, oft in Abhängigkeit vom Primärtumor. Klinisch handelt es sich um kutan/subkutan rasch wachsende Tumoren von mäßiger bis fester Konsistenz, plaque-artige Infiltrationen und granulomatöse Tumoren. Die Nichtbeteiligung von Epidermis und die freie subepidermale Dermis sprechen für eine kutane Metastase und gegen einen primären Hauttumor. Die Klärung wird in vielen Fällen durch spezielle tumor-typische Marker erreicht. Wir haben zwischenzeitlich eine große Zahl gut definierter immunhistochemischer Marker für Zell- und Tumortypen zur Verfügung, die entscheidend dazu beitragen, den Primärtumor mehr oder weniger einzugrenzen oder gar zu identifizieren. Dadurch werden unseren Patienten viele Untersuchungen - teils auch invasive - erspart.
Abstract

Next to skin tumors, dermatologic oncology also includes metastases of carcinomas of internal organs whose diagnosis has important implications for patient's management and prognosis. In the present paper we summarize clinical features, histopathology and immunohistochemical differential diagnosis of cutaneous metastases. $2-5 \%$ of malignant tumors give rise to skin metastases, mostly - but not always - in advanced tumor stages. The must frequent primary tumor is breast cancer (in females), followed by cancers of lung, colon and rectum, ovary, malignant melanoma, carcinoma of head and neck, kidney and stomach. Cutaneous metastases preferentially localize to scalp, thorax and umbilical region, often in correlation to the primary tumor. Clinically they appear as cutaneous/subcutaneous, rapidly growing nodules of medium consistency, but plaque-like infiltrations or granulomatous tumors, also occur. Epidermis and upper dermis usually are not involved in cutaneous metastases, in contrast to primary skin tumors. Immunohistochemical tumor markers are valuable in the clarification of the diagnosis. Meanwhile a large spectrum of well-defined markers for cell and tumor type are available and may be very helpful in narrowing down or even identifying the primary tumor. Thus, laborious clinical investigations may be saved. 
Einleitung

Ein wichtiges Teilgebiet der Dermatologie in der täglichen Praxis sind die Hauttumoren. Neben einer Vielfalt epidermaler, adnexabgeleiteter, melanozytärer oder mesenchymaler Tumoren sollte auch an die Möglichkeit einer Hautmetastase eines Malignoms innerer Organe gedacht werden. Für die Therapie und Prognose ist dies von entscheidender Bedeutung.

In dieser kurzen Übersicht sollen Klinik und Histopathologie gegenübergestellt werden [1]. Besonderes Gewicht kommt dabei der immunhistochemischen Differenzialdiagnose zu, für die eine zunehmende Zahl gut definierter Marker für den Zell- und Tumortyp zur Verfügung stehen. Durch die Synopsis von klinischem Befund, konventioneller Histopathologie und immunohistochemischem Markerprofil lässt sich der Primärtumor mehr oder weniger eingrenzen oder sogar identifizieren.

\section{Klinik und Epidemiologie}

Jedes Malignom kann prinzipiell in die Haut metastasieren, was allerdings nur in 2-5\% aller Fälle geschieht. Dies ist relativ selten, bedenkt man, dass die Haut das größte Organ des Menschen ist und eine sehr gute Blutversorgung aufweist. Die Metastasierungswege sind hämatogen oder lymphogen. Die hämatogene Metastasenbildung verläuft in der Regel über eine Lungenpassage, manchmal über spezielle venöse Verbindungen. Die lymphogene Metastasierung erfolgt retrograd [2 - 5]. Bemerkenswerterweise stimmt die Inzidenz der Metastasen nicht mit der der Primärtumoren überein. Am häufigsten (ca. 2/3 aller Fälle) treten Hautmetastasen bei Patientinnen mit einem Mammakarzinom auf. Es folgen - bei beiden Geschlechtern - das Lungenkarzinom, das kolorektale Karzinom, das maligne Melanom, das Kopf-HalsKarzinom, das Nierenzell-Karzinom und das Magenkarzinom. Bei Frauen ist noch das Ovarialkarzinom häufig [2,3].

Üblicherweise stellen kutane Metastasen ein spätes Ereignis im Verlauf der Tumorkrankheit dar und sind ein Zeichen für eine kurze Überlebenszeit des Patienten, die meist nur noch wenige Monate beträgt. Allerdings können kutane Metastasen auch Erstmanifestation eines Malignoms sein, insbesondere bei Primärtumoren der Lunge, der Niere und des Ovars [2,3,6]; dann ist naturgemäß die histopathologische Differenzialdiagnostik von großer Bedeutung für die Identifikation des Primärtumors.

Generell ist die Lokalisation an der Haut nicht arbiträr, sondern es bestehen Prädilektionsstellen, die vom Primärtumor abhängig sind. Die Rumpfhaut ist oft betroffen; hier sind Mammakarzinom-Metastasen besonders häufig, für die ein lymphogener Metastasierungsweg angenommen wird. Die Nabelregion ist häufig von kutanen Metastasen befallen, insbesondere bei abdominellen Karzinomen, die dann Schwester-Mary-Joseph-Knoten genannt werden. Auch die Kopfhaut ist ein häufiger Metastasierungsort (bis zu $13 \%$ aller Hautmetastasen), wahrscheinlich wegen ihrer guten Vaskularisation [5-7]. Besonders zu erwähnen sind Tumorabsiedelungen im Bereich von Drainage- oder Endoskopie-Kanälen und in Operationsnarben bei abdominellen oder gynäkologischen Karzinomen.
Klinisch handelt es sich meist um solitäre oder multiple, rasch wachsende Tumoren von mäßig fester Konsistenz, die selten wenn überhaupt - ulzerieren. Seltener sind Plaque-artige Infiltrationen und granulomatöse Tumoren.

Aufgrund der uncharakteristischen Klinik kann die Diagnose in der Regel erst histopathologisch gestellt werden. In der üblichen HE-Routinefärbung sprechen generell eine Nichtbeteiligung der Epidermis und Adnexe und eine freie subepidermale Dermis für eine kutane Metastase und gegen einen primären Hauttumor. Eine Klärung wird insbesondere durch die Immunhistochemie mit speziellen Tumor-typischen Markern erreicht.

\section{Adenokarzinome}

Sie machen mehr als $60 \%$ aller Hautmetastasen aus und können von vielen Organen ausgehen. Ihre Zuordnung zum jeweiligen Primärtumor bzw. Ursprungsorgan kann sehr schwierig sein $[8,9]$. Bei der Frau verursachen Mammakarzinome die Mehrzahl aller kutanen Metastasen überhaupt (Abb.1a). Beim häufigsten Typ, dem invasiven duktalen Mammakarzinom, herrschen in der Dermis proliferierte, kohäsive Tumorzellverbände aus soliden Trabekeln und Tubuli vor. Das Stroma kann unterschiedlich ausgeprägt sein, auch desmoplastische Stromabildungen kommen vor, die ein Mammakarzinom vermuten lassen (Abb.1b; [4-6,9,10]). Die Diagnose eines Mammakarzinoms kann an der Metastase immunhistochemisch bestätigt werden, und zwar durch den Nachweis des typischen apokrinen Markers, Gross Cystic Disease Fluid-Protein-15 (GCDFP-15), der in bis zu $85 \%$ der Fälle von Mammakarzinomen positiv ist [11,12] und durch den Nachweis des Östrogenrezeptors, der in bis zu $75 \%$ der Fälle positiv ist (Abb.1 c; [12,13]). Beide Marker zusammen ergeben eine hohe Sensitivität [14]. Schwierig kann die Abgrenzung von Schweißdrüsenkarzinomen sein, die häufig diese beiden Marker ebenfalls exprimieren. Hier kann die Expression des epidermalen Wachstumsfaktor-Rezeptors (EGF-R) weiterhelfen, dessen Positivität mehr für ein Schweißdrüsenkarzinom spricht [13]. Invasive duktale Mammakarzinome sind positiv für das epitheliale Zelladhäsionsmolekül E-Cadherin [15].

Kutane Metastasen des invasiven lobulären Mammakarzinoms sind histopathologisch charakterisiert durch in der Dermis verstreut wachsende Tumorzellen zwischen den Kollagenfasern (Abb. 2a). Die Stromadesmoplasie ist sehr variabel; in sehr ausgeprägten Fällen finden sich nur wenige vereinzelte Tumorzellen disseminiert im Bindegewebe [16]. Dann kann es schwierig sein, überhaupt einen Tumor zu erkennen und von einer chronischen Entzündung oder einem mesenchymalen Tumor abzugrenzen. Auch hier helfen immunhistochemische Färbungen entscheidend weiter. Tumorzellen des Mammakarzinoms sind generell Keratin-positiv, und beim invasiven lobulären Karzinom sind Keratin-Antikörper insbesondere in der Lage, auch einzelne Tumorzellen nachzuweisen (Abb. 2 b). Auch hier können die Expression von GCDFP-15 und Östrogen-Rezeptoren die Herkunft von der Mamma bestätigen. Fälle mit einer massiven Stroma-Wucherung werden klinisch Cancer en cuirasse [6] genannt, dessen Charakteristikum sklerodermiforme flächenhafte Hautinfiltrate der Brustregion sind. Das invasiv lobuläre Mammakarzinom ist - passend zu dem verstreuten Wachstumsmuster - negativ für 

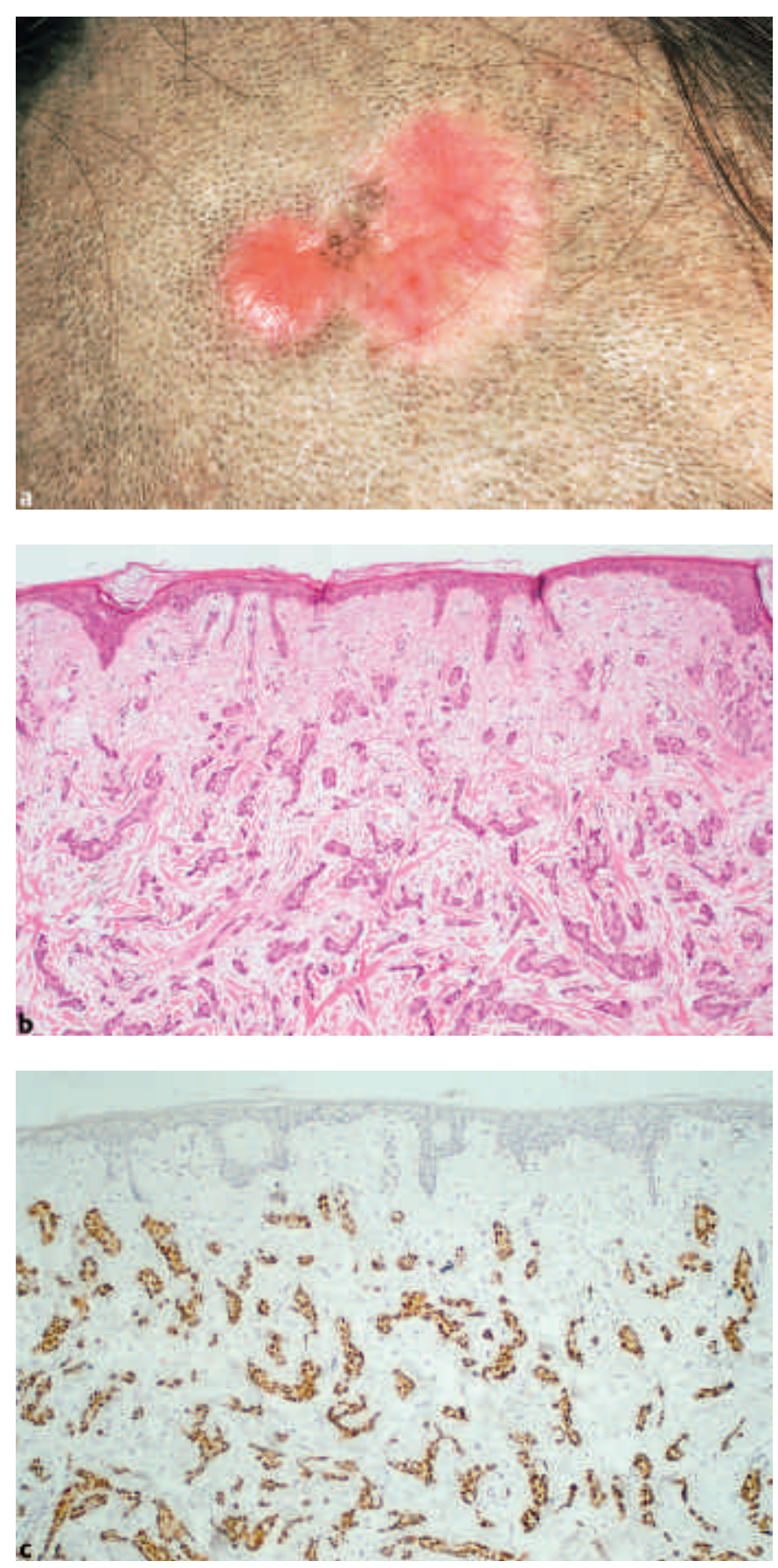

Abb. 1 Hautmetastasen eines Mammakarzinoms (Frau, parietal links, 70 J.). Zwei erythematöse, derbe Plaques, Metastase (a) eines mäßig differenzierten invasiven duktalen Mammakarzinoms mit irregulären trabekulären und tubulären Zellverbänden in der Dermis (b; HE-Färbung). Immunhistochemisch reagieren die Tumorzellkerne stark mit einem Antikörper gegen den Östrogen-Rezeptor (ABC-Peroxidase-Färbung; $\mathbf{c}$.

das Zelladhäsionsmolekül E-Cadherin, was für die Abgrenzung gegenüber dem invasiven duktalen Mammakarzinom hilfreich sein kann [15,16]. Magenkarzinome des diffusen Typs können dem invasiven lobulären Mammakarzinom histopathologisch ähnlich sein und somit eine nicht seltene histologische Differenzialdiagnose darstellen. Erstere sind aber oft - im Gegensatz zum Mammakarzinom - positiv für Keratin 20 (s.u.), während die mammatypischen Marker fehlen.
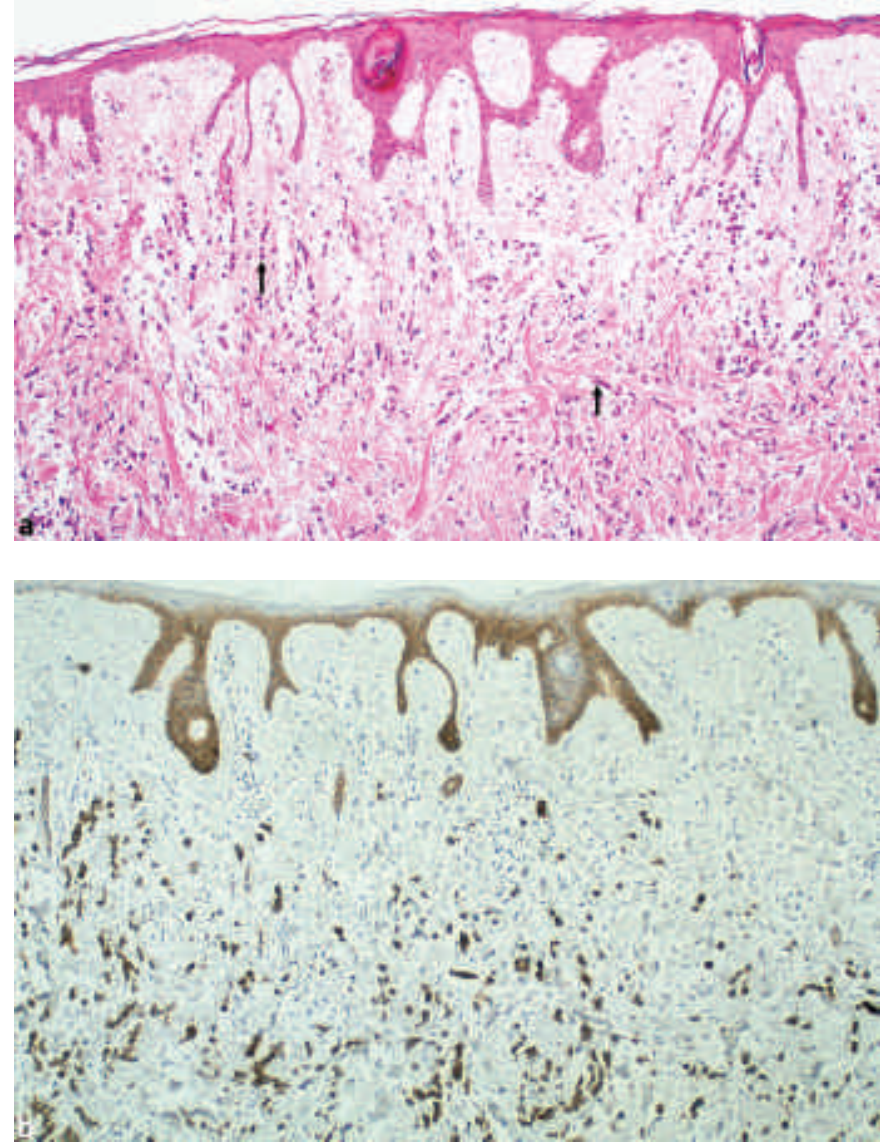

Abb. 2 Hautmetastase eines invasiven lobulären Mammakarzinoms (Frau, Abdomen). Disseminierte unscheinbare Tumorzellen in der Dermis mit Ausbildung gänsemarschartiger Reihen (a; Pfeile; HE-Färbung). (b) Die disseminierten Tumorzellen sind positiv für pan-Keratin (Klon MNF 116).

Lungenkarzinome, die zweithäufigste Quelle von kutanen Metastasen und relativ oft deren Erstmanifestation [18], ähneln klinisch den Hautmetastasen anderer rasch eruptiver Tumoren, z. B. einem Keratoakanthom (Abb. 3). Die Hautmetastasen von pulmonalen Adenokarzinomen sind histopathologisch heterogen, können azinär, papillär oder solid aufgebaut sein und sind häufig schlecht differenziert. Dann sind Marker, vor allem der thyreoidale Transkriptions-Faktor-1 (TTF-1) sehr hilfreich, der in bis $95 \%$ der Fälle [17 - 19] positiv ist, während Keratin 20 meist fehlt (s.u.).

Auch das kolorektale Karzinom ist häufig Ursache von Hautmetastasen, die meist am Abdomen, im Nabelbereich, aber auch im Gesicht-Hals-Bereich und am behaarten Kopf lokalisiert sind. Charakteristisch sind Adenokarzinom-Infiltrate von unterschiedlicher Differenzierung (Abb. 4a); Nekrosen können hinzukommen $[2,3,5]$. Auch muzinöse Formen kommen vor. Am hilfreichsten in der Abklärung sind die Positivität für Keratin 20 (Abb. 4b) und das Fehlen von Keratin 7 (Tab.1; [20-22]).

Adenokarzinome des Magens metastasieren bevorzugt am Stamm mit einem variablen klinischen Erscheinungsbild. Mikroskopisch kann man einen intestinalen und einen diffusen Typ unterscheiden ([23], Abb.5a,b). Die typische Koexpression von 


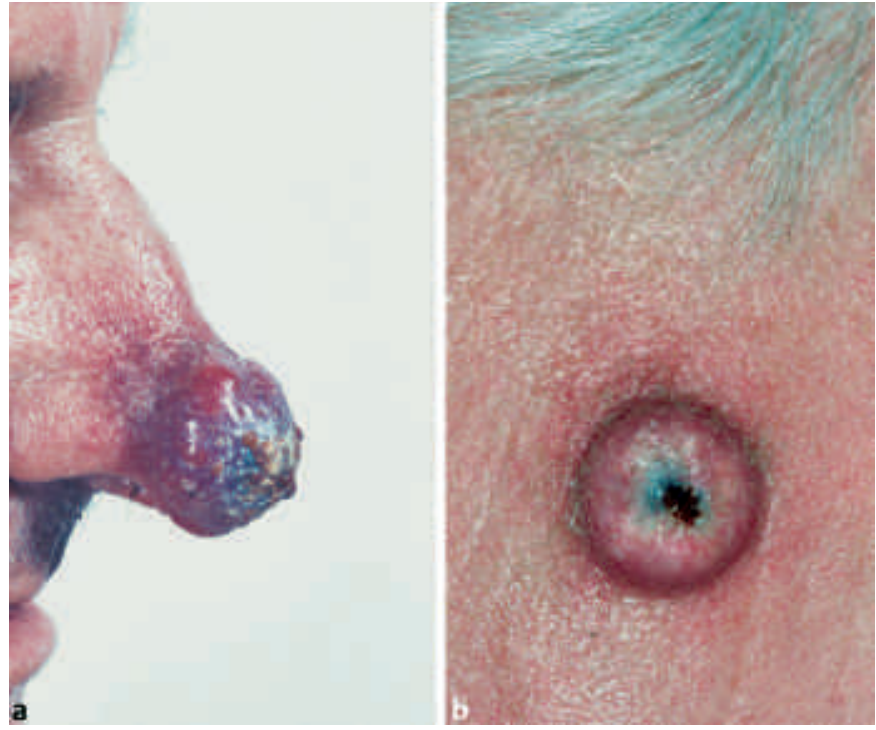

Abb. 3 (a) Hautmetastase eines Adenokarzinoms der Lunge, ein rasch wachsender bläulich-livider zentral ulzerierter Tumor; (b) zentral ulzeriertes Keratoakanthom.

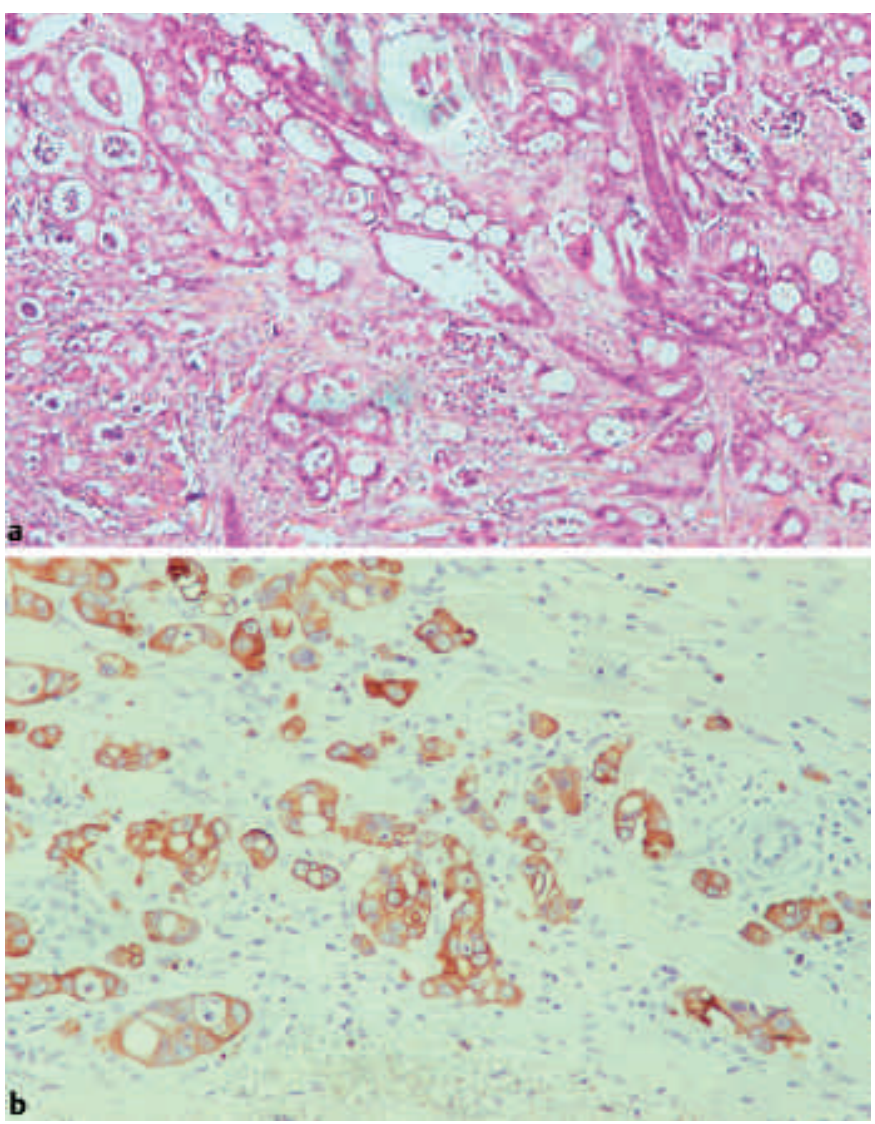

Abb. 4 Kutane Metastase eines Adenokarzinoms des Kolons (behaarter Kopf). (a) Infiltrate mit adenoider und muzinöser Differenzierung in der Dermis (HE-Färbung; b) Immunhistochemisch ausgeprägte Reaktion der Tumorzellen für Keratin 20, das typisch für gastrointestinale Epithelien ist.

Keratin 20 (Abb. 5c) und Keratin 7 bei Abwesenheit von TTF-1, GCDFP-15 und Östrogenrezeptoren weist immunhistochemisch auf ein Magenkarzinom hin und erlaubt die Differenzierung von

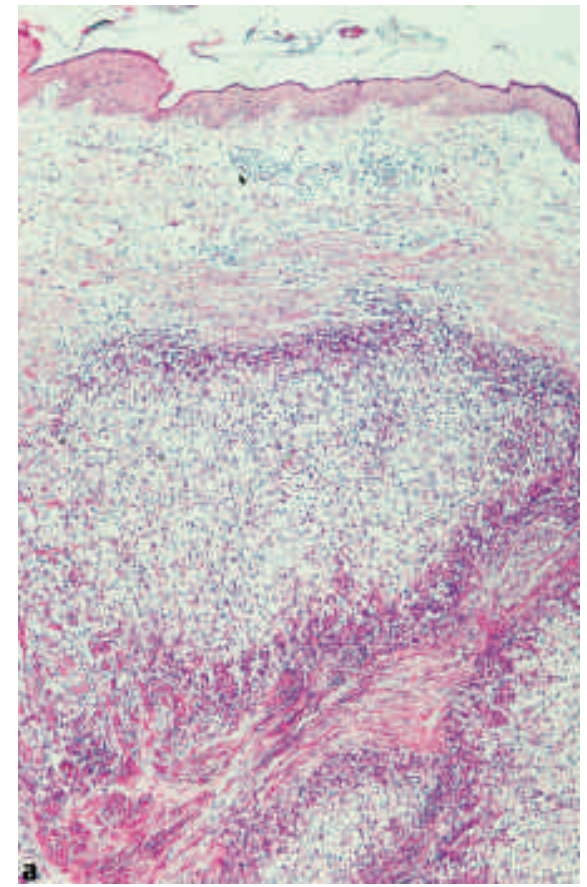

Abb. 5 Hautmetastase eines Adenokarzinoms des Magens (Abdomen). Siegelring-Zellinfiltrate in der Dermis (a, b; HEFärbung). Immunhis tochemisch starke Anfärbung der Tumorzellen mit Keratin 20-Antikörper (Klon KS 20.8; c).

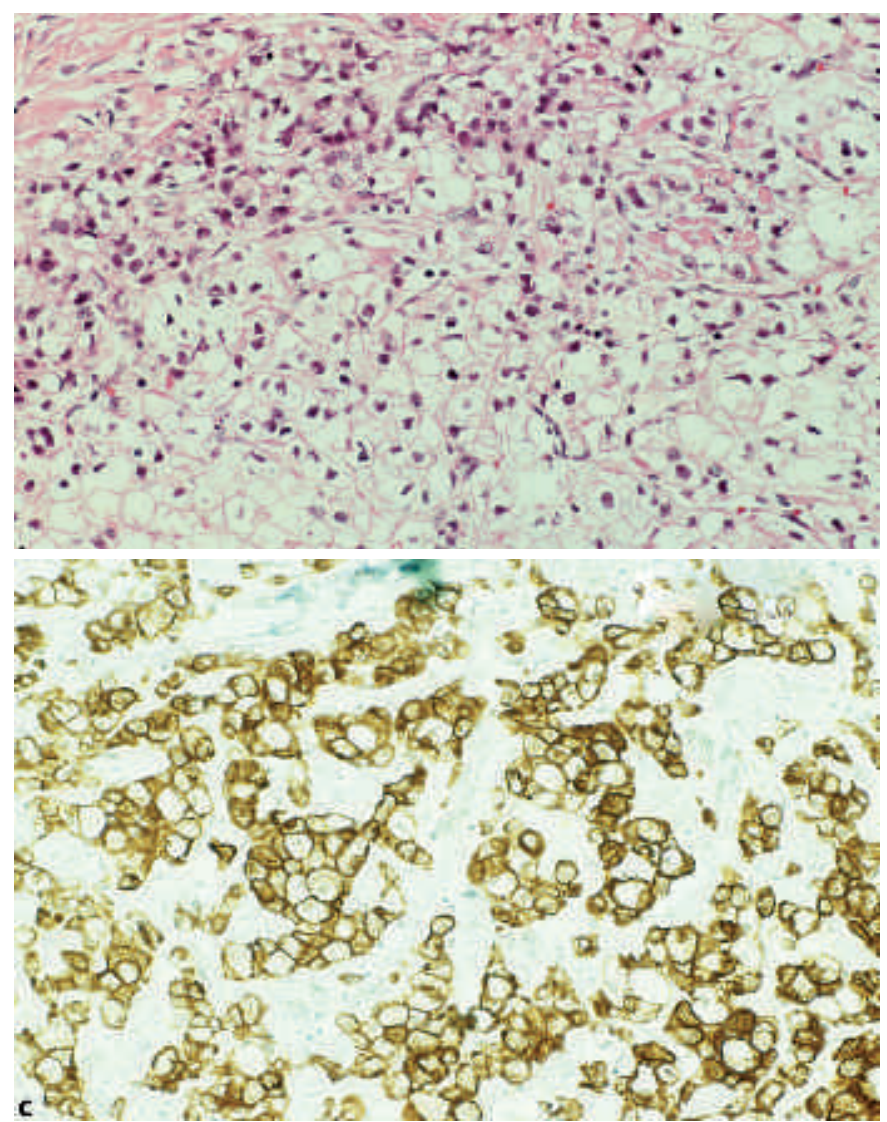

kolorektalen und pulmonalen Adenokarzinomen und von Mammakarzinomen (s. Tab. $\mathbf{1} ;[5,8,14,19,22])$.

Hautmetastasen bei Ovarialkarzinomen sind selten, treten erst im späten Stadium mit Peritonealkarzinose auf, nur gelegentlich als Erstmanifestation. Sie lokalisieren bevorzugt in der Nabelregion und im Bereich von Operationsnarben (Abb. 6a). Papilläre 
Tab. 1 Marker in der Diagnostik von Hautmetastasen

\begin{tabular}{|llllllll}
\hline & \multicolumn{1}{c}{ CK7 CK20 CK5 CCDFP-15 ER } \\
\hline
\end{tabular}

1 Östrogenrezeptor

* neuroendokrine Marker (Synaptophysin, CgA, NCAM u.a.) positiv

** Lymphom-Marker (CLA) positiv

*** Melanom-Marker (HMB45, Melan A, S 100) positiv.

Strukturen (Abb.6b) und kalzifizierte Psammom-Körperchen sind typisch für den serös-papillären Subtyp des Ovarialkarzinoms $[6,20,24,25]$. Immunhistochemisch kann die Bestimmung der Intermediärfilamente - Keratin 7 positiv, Keratin 20 negativ, Vimentin häufig positiv - gemeinsam mit der Positivität für CA-125 (Abb. 6c) auf ein primäres Ovarialkarzinom hinweisen $[24,25]$.

Hautmetastasen des Adenokarzinoms der Prostata sind sehr selten, trotz der hohen Inzidenz dieses Malignoms. Es herrschen am unteren Abdomen und am behaarten Kopf mäßig derbe, rötlich-violette Knoten vor. Histopathologisch haben die Adenokarzinome der Prostata oft einen kleindrüsigen oder kribriformen Aufbau, die Zellen ein helles Zytoplasma und prominente Nucleoli $[4,5]$. Wesentlich für die Zuordnung zur Prostata ist aber der immunhistochemische Nachweis des Prostata-spezifischen Antigens (PSA).

\section{Klarzellige Karzinome}

Klarzellige Hautmetastasen eines primären Nierenzellkarzinoms sind relativ häufig und können auch Erstmanifestation oder Spätmetastase sein [2-5,26]. Sie sind bevorzugt am Kopf oder Hals lokalisiert und livide bläulich verfärbt (Abb. 7). Wie beim Primärtumor erscheinen histopathologisch die Zellen der Hautmetastasen durch herausgelöstes Glykogen und Lipide optisch leer. Die klinische und histopathologische Differenzialdiagnose umfasst hell- und klarzellige Karzinome anderer Organe, vornehmlich Leber, Schilddrüse, Speicheldrüse, Nebennierenrinde und Ovar, aber auch klarzellige primäre Hauttumoren wie Hidradenome, Talgdrüsentumoren oder eine seltene Variante von Basalzellkarzinomen [4-6]. Diese Hauttumoren weisen praktisch immer - zumindest partiell - Keratin 5 auf, was differenzialdiagnostisch verwendet werden kann. Hingegen zeichnen
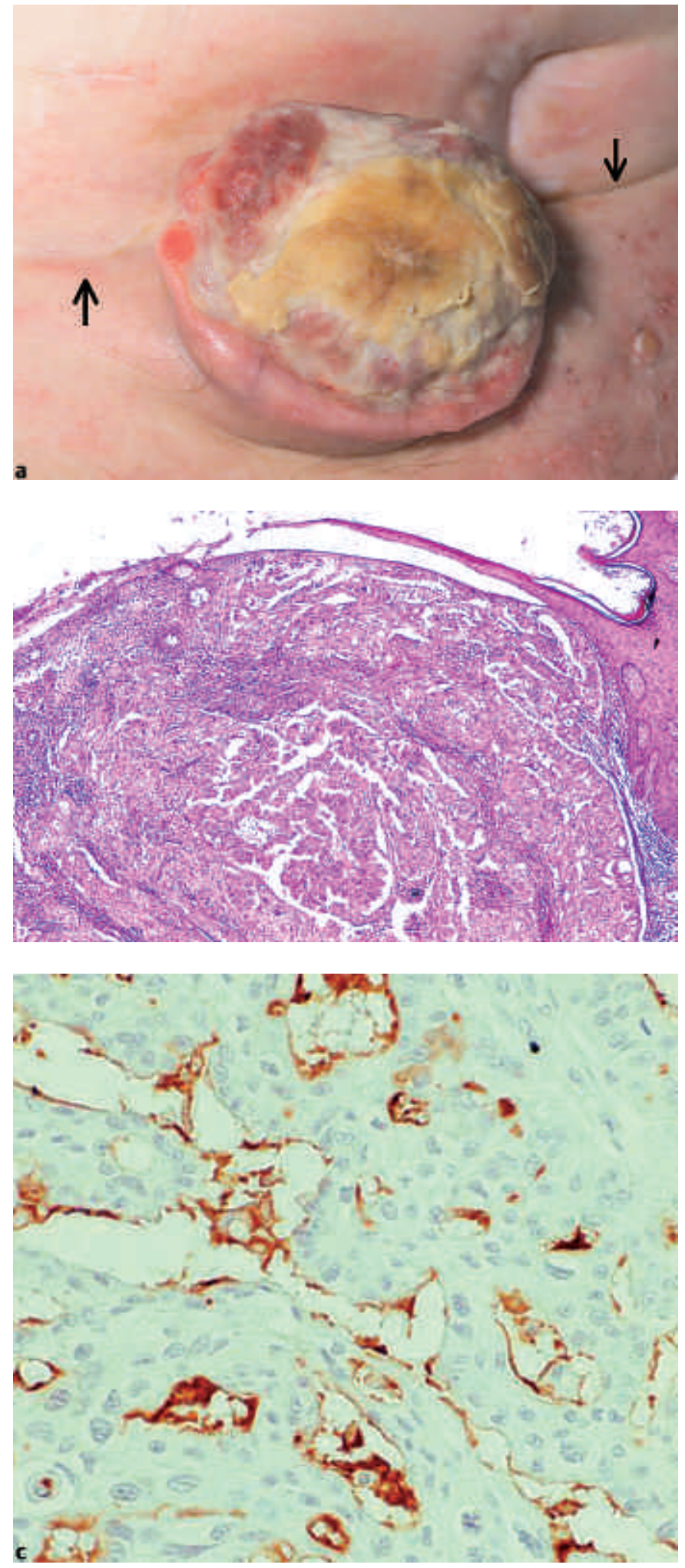

Abb. 6 Hautmetastase eines Ovarialkarzinoms (Abdomen) in der Operationsnarbe (a; Pfeile). Papilläre Tumorinfiltrate in der Dermis, (b; E Epidermis; HE-Färbung). Immunhistochemisch starke Positivität der Tumorzellen für CA 125 (c).

sich klarzellige Nierenzellkarzinome durch die relativ seltene Koexpression von Keratin 8/18 mit Vimentin aus, hinzu kommt der Nachweis von CD 10 [26]. 


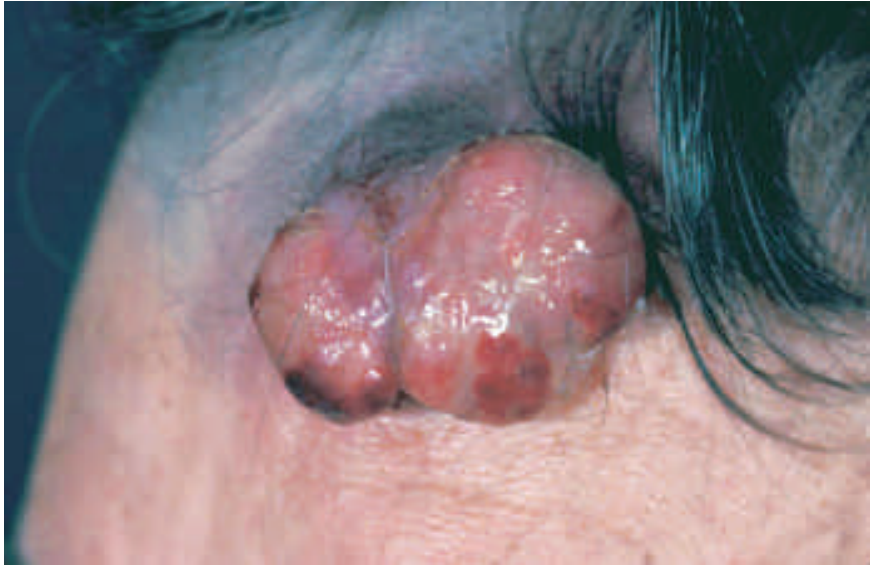

Abb. 7 Hautmetastase eines Nierenzellkarzinoms. Granulomatösfleischiger Knoten (Mann, 68 J.; Haaransatz, li.).
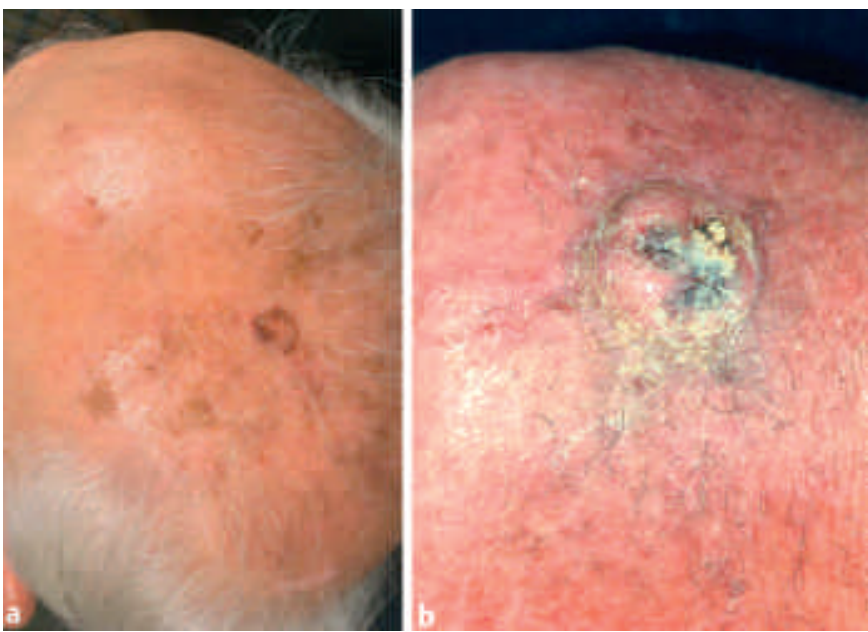

Abb. 8 (a) Multiple aktinische Keratosen (77 J.). (b) Noduläres zentral exulzeriertes Spinaliom (78 J.; in chronisch lichtgeschädigter Haut [Scheitel]).

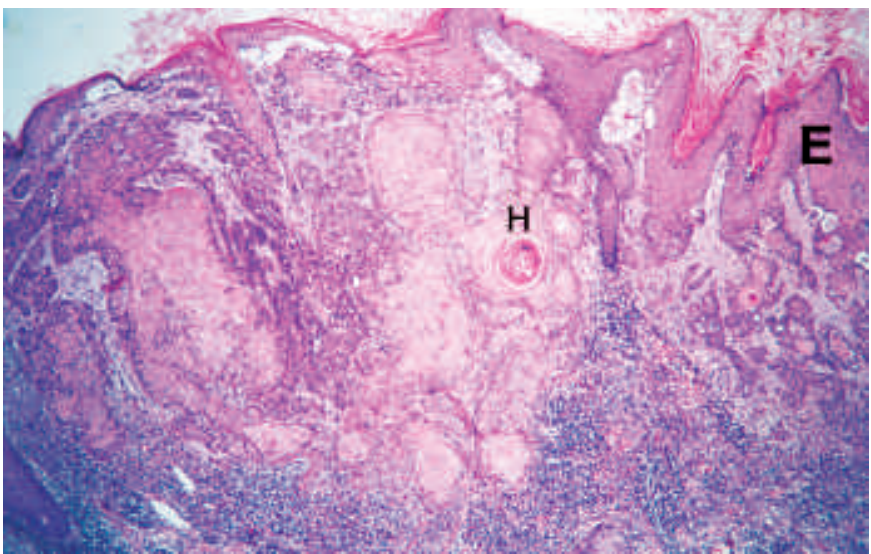

Abb. 9 Spinaliom mit karzinomatösen Plattenepithelzapfen, die von der Epidermis (E) in die Dermis einwachsen in mäßiger Differenzierung mit Ausbildung von Hornperlen (H). HE-Färbung.
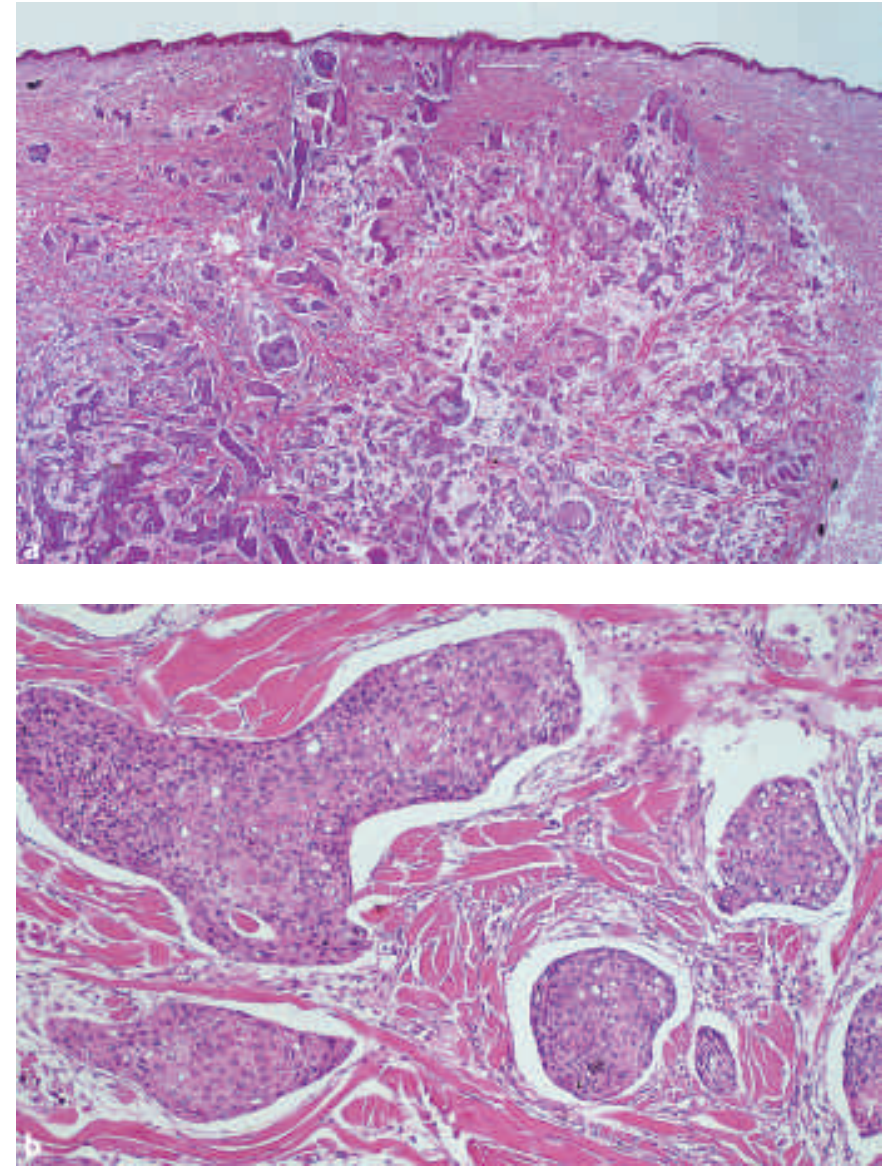

Abb. 10 Hautmetastase eines Plattenepithelkarzinoms des Oropharynx mit freier subepidermaler Zone (Schulterregion; a), anastomosierende solide Nester und Trabekel des Plattenepithelkarzinoms in der Dermis (b). HE-Färbung.

\section{Plattenepithelkarzinome}

Bei plattenepithelialen malignen Hautinfiltraten besteht immer die Schwierigkeit der Abgrenzung einer Hautmetastase eines inneren Plattenepithelkarzinoms von einem Spinaliom. Typisch für Spinaliome ist deren Lokalisation an der lichtexponierten Haut des Gesichtes, Scheitels, Handrückens und der Unterarme sowie der distalen Unterschenkel und ihre häufige Entstehung aus aktinischen Keratosen, die meist noch randständig nachweisbar sind. Hinzu kommen die aktinischen Schädigungen der Umgebungshaut wie Epidermisatrophie mit Rarifizierung der Leisten und aktinische Elastose in der Dermis (Abb. 8a,b). Die Verbindung mit der Epidermis und die von hier ausgehende Invasion sind deutlich ([4,5]; Abb. 9). Im Gegensatz dazu sind Plattenepithelkarzinom-Metastasen bevorzugt in der Dermis ohne Verbindung zum Epithel lokalisiert (Abb. 10). Als Primärtumoren kommen eine Reihe von Organen wie Mund, Rachen, Kehlkopf, Ösophagus, Lunge und Cervix uieri in Frage. Obwohl bei Lungenkarzinomen das Plattenepithelkarzinom der häufigste histologische Subtyp ist, bildet dieses im Vergleich zu den Adenokarzinomen (s.o.) weniger häufig Hautmetastasen [4,5,9]. Nur selten metastasieren Plattenepithelkarzinome des Kopf-Hals-Bereichs und des Ösophagus in die Haut, meist erst in fortgeschrittenen Stadien der Tumorkrankheit. Bei den Kopf-Hals-Tumoren sind die Metastasen oft in der Kopf- oder Halshaut lokalisiert. Histo- 


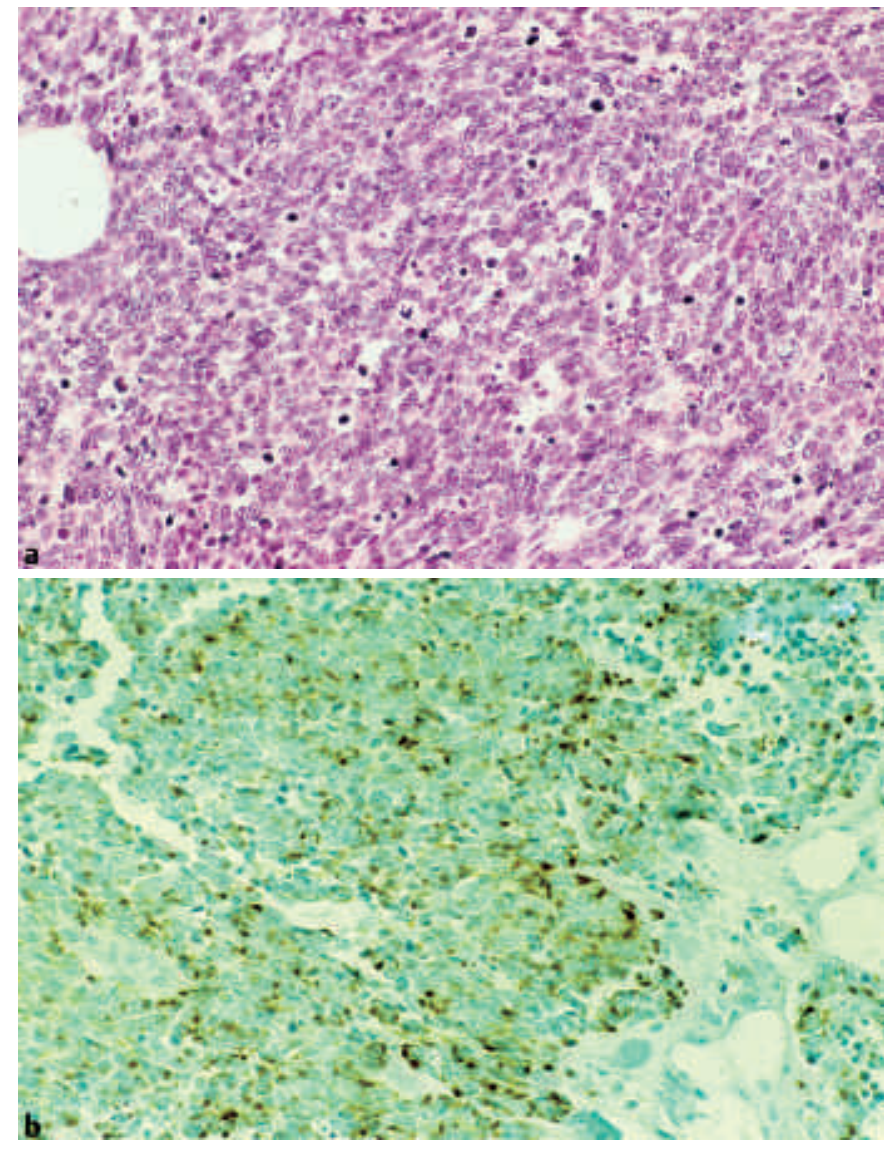

Abb. 11 Hautmetastase eines kleinzelligen Bronchialkarzinoms (a; HE-Färbung). Immunhistochemisch färben Antikörper gegen Keratin 18 (Klon Ks 18.04) vorwiegend plaque-förmige Strukturen in den Tumorzellen (b).

pathologisch dominieren schlecht differenzierte Plattenepithelkarzinomverbände in der Dermis [27]. Immunhistochemisch lassen sich generelle Plattenepithelmarker wie Keratin 5 nachweisen, die bei schlechter Differenzierung den Plattenepithelcharakter aufzeigen, aber nicht zwischen Metastasen verschiedener Herkunft und Spinaliomen unterscheiden. Hinsichtlich des spezifischen Ausgangsorgans sind keine wegweisenden Tumormarker bekannt. Wenn wahrscheinlich ist, dass es sich um eine Hautmetastase handelt, muss der Primärtumor unter den o.g. Organen klinisch gesucht werden.

\section{Kleinzellige/neuroendokrine Karzinome}

Bei diesen Metastasen handelt es sich überwiegend um Hautmetastasen des kleinzelligen Bronchialkarzinoms. Sie sind oft an der Haut des Körperstamms lokalisiert. Verglichen mit der Häufigkeit und Metastasierungsneigung des kleinzelligen Bronchialkarzinoms sind Hautmetastasen dieses Tumors eher gering. Histopathologisch handelt es sich um solide, kleinzellige, undifferenzierte Tumorzellverbände mit rundlichen bis ovalären Zellkernen und sehr wenig Zytoplasma (Abb.11a). Das kleinzellige Bronchialkarzinom wird immunhistochemisch markiert durch die niedermolekularen Zytokeratine Keratin 8 und 18, während Keratin 20 meist negativ ist (Abb.11 b). Hinzu kommen die neuroendokrinen Marker Synaptophysin und CD 56 (N-CAM) sowie

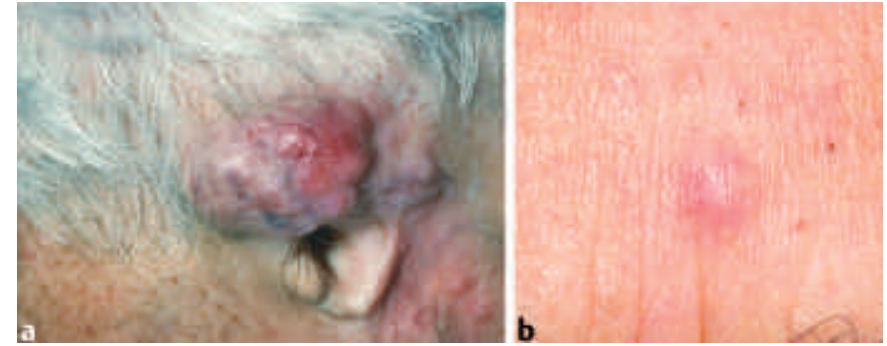

Abb. 12 Merkelzellkarzinom. Bläulich-rötlich livide Lokalrezidive, deren Operationen und Destruktionen das Ohr zerstörten (a; Mann, 93 J.). (b) Subkutane Metastase (Stirn; Mann, 62 J.).

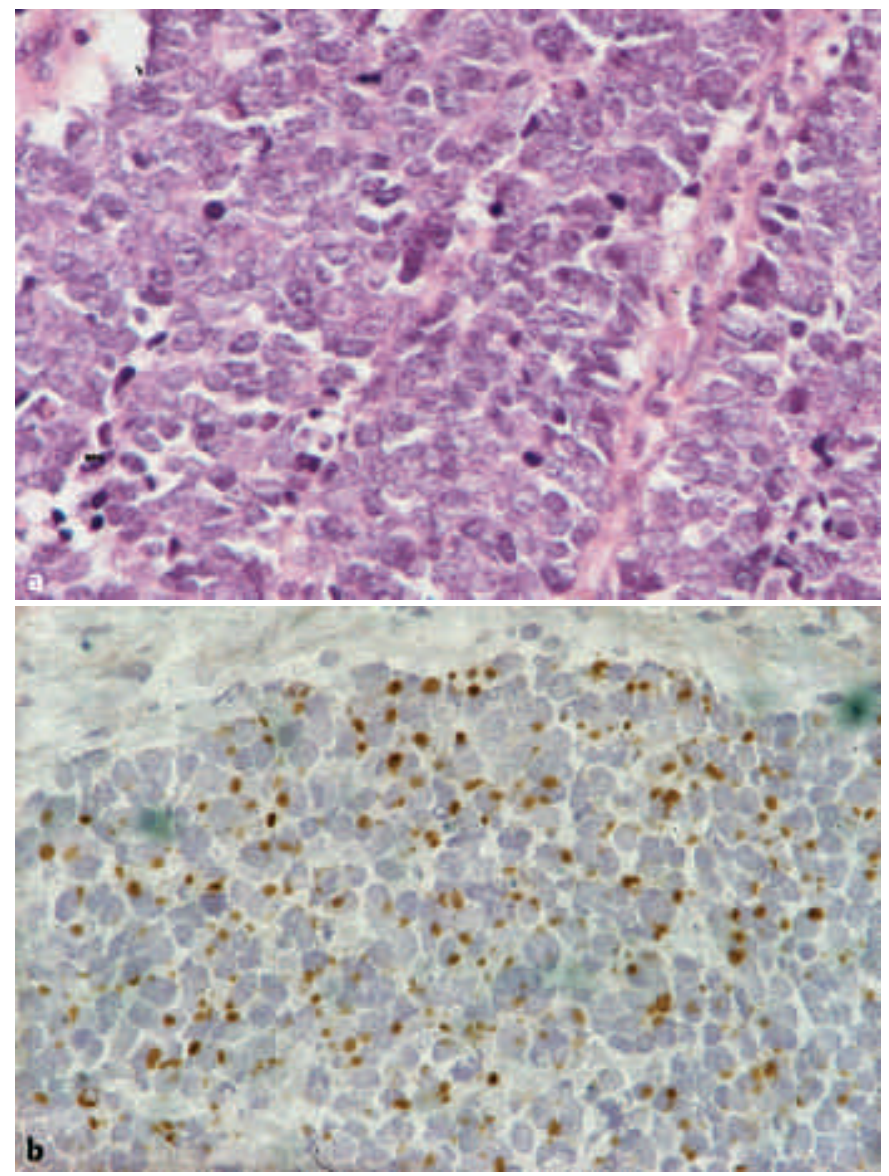

Abb. 13 Merkelzellkarzinom. Homogene, wenig differenzierte Tumorzellen in der Dermis, kleinzelliger Zelltyp (a). Immunhistochemisch deutlich positiv mit Antikörpern gegen Keratin 20 (Klon Ks 20.8) in einem Punktmuster durch Färbung der paranukleären Plaques (b).

TTF-1 $[17,19,21,28]$. Die Differenzialdiagnose schließt verschiedene primäre Hauttumoren, vor allem das Merkelzellkarzinom, ein. Diese Tumoren und ihre Hautmetastasen sind klinisch (Abb.12) oft ähnlich und auch die Routine-Histologie ermöglicht meist keine eindeutige Abgrenzung (Abb.13a). Die sehr charakteristische Koexpression von niedermolekularen Keratinen, insbesondere Keratin 20 (Abb.13 b), mit Neurofilament-Protein erlaubt die Diagnose eines Merkelzellkarzinoms und seine Abgrenzung vom kleinzelligen Bronchialkarzinom [29,30]. Neuronale und neuroendokrine Marker wie Chromogranin, Synaptophysin und N-CAM können hingegen in beiden Tumortypen exprimiert werden [4]. Problematisch kann in der Routinehistologie die Ab- 
grenzung maligner Lymphome von kleinzelligen Hautmetastasen und Merkelzellkarzinomen sein. Erstere exprimieren charakteristischerweise Leucocyte Common Antigen (CD 45) und B-Zell- bzw. T-Zell-Marker, während Keratine fehlen ([4,5]; Tab. 1).

\section{Maligne Melanome}

Hautmetastasen von malignen Melanomen sind relativ häufig. Sie können sowohl In-Transit-Metastasen als auch hämatogene Fernmetastasen darstellen, die charakteristischerweise einen Epidermotropismus zeigen, der bei Hautmetastasen anderer Primärtumoren meist fehlt. Der Epidermotropismus kann daher bei kutanen Infiltraten maligner Melanome auch kein spezifisches Kriterium für ein primäres Melanom sein. Makroskopisch sind die Metastasen melanotisch oder amelanotisch und in unterschiedlichen Hautschichten lokalisiert. Immunhistochemisch sind sie typischerweise negativ für Keratine und positiv für Vimentin und verschiedene melanozytäre Marker wie HMB 45, Melan A und S $100[4,5,31]$.

\section{Schlussfolgerungen}

Hautmetastasen von Karzinomen innerer Organe sind selten und meist Zeichen eines Spätstadiums. Sie sind Ausdruck einer Fernmetastasierung, was das therapeutische Procedere entscheidend bestimmt. In manchen Fällen sind sie aber auch die Primärmanifestation eines Malignoms, und dann ist ihre Diagnostik wegweisend für die Identifikation des Primärtumors, der in diesen Fällen häufig in der Lunge, der Niere oder im Ovar lokalisiert ist. Immunhistochemische Marker, die inzwischen in einer breiten Palette und gut charakterisiert zur Verfügung stehen, erlauben bei Hautmetastasen Rückschlüsse auf den Primärtumor. Ihr Einsatz lohnt sich in der Histodiagnostik, da die Primärtumoren damit auf einen oder wenige eingeengt werden und den Patienten so oft weitere Untersuchungen erspart werden können. Besondere Bedeutung hat die Abgrenzung einer Metastase von einem primären Hauttumor.

\section{Literatur}

${ }^{1}$ Moll R, Moll I. Hautmetastasen. In: Remmele W (Hrsg). Pathologie (3. Auflage), Kap. 17, Berlin: Springer, im Druck, 2005

2 Brownstein MH, Helwig EB. Metastatic tumors of the skin. Cancer 1972; 29: $1298-1307$

${ }^{3}$ Brownstein MH, Helwig EB. Spread of tumors to the skin. Arch Dermatol 1973; 107: 80-86

${ }^{4}$ Hödl S, Kerl H. Hautmetastasen viszeraler Karzinome. In: Kerl H, Garbe C, Cerroni L, Wolff HH (Hrsg). Histopathologie der Haut (Kap. 41). Berlin: Springer, 2003: 925 -941

${ }^{5}$ Weedon D. Skin pathology. Cutaneous metastases. London: Churchill Livingstone, 2002: 1045-1055

${ }^{6}$ Schwartz RA. Cutaneous metastatic disease. J Am Acad Dermatol 1995; 33: $161-182$

${ }^{7}$ Krathen RA, Orengo IF, Rosen T. Cutaneous metastasis: a meta-analysis of data. South Med J 2003; 96: 164-167

${ }^{8}$ Saikia B, Dey P, Saikia UN, Das A. Fine needle aspiration cytology of metastatic scalp nodules. Acta Cytol 2001; 45: 537-541
${ }^{9}$ Lookingbill DP, Spangler N, Helm KF. Cutaneous metastases in patients with metastatic carcinoma: a retrospective study of 4020 patients. J Am Acad Dermatol 1993; 29: 228 -236

${ }^{10}$ Lookingbill DP, Spangler N, Sexton FM. Skin involvement as the presenting sign of internal carcinoma. J Am Acad Dermatol 1990; 22: $19-26$

${ }^{11}$ Le Doussal V, Zangerle PF, Collette J, Spyratos F, Hacene K, Briere M, Franchimont P, Gest J. Immunhistochemistry of a component protein of the breast cystic disease fluid with mol. wt 15,000. Eur J Cancer Clin Oncol 1985; 21: 715-725

12 Wick MR, Ockner DM, Mills SE, Ritter JH, Swanson PE. Homologous carcinomas of the breast, skin, and salivary glands. A histologic and immunohistochemical comparison of ductal mammary carcinoma, ductal sweat gland carcinoma, and salivary duct carcinoma. Am J Clin Pathol 1998; 109: 75-84

${ }^{13}$ Busam KJ, Tan LK, Granter SR, Kohler S, Junkins-Hopkins J, Berwick M, Rosen PP. Epidermal growth factor, estrogen, and progesterone receptor expression in primary sweat gland carcinomas and primary and metastatic mammary carcinomas. Mod Pathol 1999; 12: 786-793

${ }^{14}$ Ormsby AH, Snow JL, Su WP, Goellner JR. Diagnostic immunohistochemistry of cutaneous metastatic breast carcinoma: a statistical analysis of the utility of gross cystic disease fluid protein-15 and estrogen receptor protein. J Am Acad Dermatol 1995; 32: 711 - 716

${ }^{15}$ Moll R, Mitze M, Frixen UH, Birchmeier W. Differential loss of E-cadherin expression in infiltrating ductal and lobular breast carcinomas. Am J Pathol 1993; 143: 1731 - 1742

${ }^{16}$ Ferlicot S, Vincent-Salomon A, Medioni J, Genin P, Rosty C, Sigal-Zafrani B, Freneaux P, Jouve M, Thiery JP, Sastre-Garau X. Wide metastatic spreading in infiltrating lobular carcinoma of the breast. Eur J Cancer 2004; 40: $336-341$

${ }^{17}$ Ambrogi V, Nofroni I, Tonini G, Mineo TC. Skin metastases in lung cancer: analysis of a 10-year experience. Oncol Rep 2001; 8: 57-61

${ }^{18}$ Schwartz RA. Histopathologic aspects of cutaneous metastatic disease. J Am Acad Dermatol 1995; 33: 649-657

${ }^{19}$ Chang YL, Lee YC, Liao WY, Wu CT. The utility and limitation of thyroid transcription factor-1 protein in primary and metastatic pulmonary neoplasms. Lung Cancer 2004; 44: 149-157

${ }^{20}$ Moll R, Löwe A, Laufer J, Franke WW. Cytokeratin 20 in human carcinomas: a new histodiagnostic marker detected monoclonal antibodies. Am J Pathol 1992; 140: 427-447

${ }^{21}$ Chu P, Wu E, Weiss LM. Cytokeratin 7 and cytokeratin 20 expression in epithelial neoplasms: a survey of 435 cases. Mod Pathol 2000; 13 : $962-972$

${ }^{22}$ Kaufmann O, Fietze E, Dietel M. Immunhistochemische Diagnostik bei Karzinommetastasen mit unbekanntem Primärtumor. Pathologe 2002; 23: 183 - 197

${ }^{23}$ Werner M, Becker KF, Keller G, Höfler H. Gastric adenocarcinoma: pathomorphology and molecular pathology. J Cancer Res Clin Oncol 2001; 127: 207-216

${ }^{24}$ Cormio G, Capotorto M, Di Vagno G, Cazzolla A, Carriero C, Selvaggi L. Skin metastases in ovarian carcinoma: a report of nine cases and a review of the literature. Gynecol Oncol 2003; 90: 682 -685

${ }^{25}$ Moll R, Pitz S, Levy R, Weikel W, Franke WW, Czernobilski B. Complexity of expression of intermediate filament proteins, including glial filament protein, in endometrial and ovarian adenocarcinomas. Hum Pathol 1991; 10: 989-1001

${ }^{26}$ Qian J, Ramnani DM, Bostwick DG. Immunohistochemistry of kidney tumors. In: Dabbs DJ. Diagnostic immunohistochemistry. New York: Churchill Livingstone, 2002: 474-485

${ }^{27}$ Yoskovitch A, Hier MP, Okrainec A, Black MJ, Rochon L. Skin metastases in squamous cell carcinoma of the head and neck. Otolaryngol Head Neck Surg 2001; 124: 248 - 252

${ }^{28}$ Sheppard MN. Specific marker for pulmonary tumor. Histopathology 2000; 36: 237-276

${ }^{29}$ Goessling W, McKee PH, Mayer R. Merkel cell carcinoma. J Clin Oncol 2002; 20: $588-598$

${ }^{30}$ Moll R, Osborn M, Hartschuh W, Moll I, Mahrle G, Weber K. Variability of Expression and Arrangement of Cytokeratin and Neurofilaments in Cutaneous Neuroendocrine Carcinomas (Merkel Cell Tumors): Immunochemical and Biochemical Analysis of Twelve Cases. Ultrastruct Pathol 1986; 10: 473-495

${ }^{31}$ Yaziji H, Gown AM. Immunohistochemical markers of melanocytic tumors. Int J Surg Pathol 2003; 11: 11 - 15 